Marcelo Medeiros ${ }^{1}$

Debora Diniz ${ }^{2}$

Ida Vanessa Doederlein Schwartz ${ }^{3}$

${ }^{1}$ Departamento de Sociologia, Universidade de Brasília. Caixa Postal 8011 70673-970 Brasília DF. anis@anis.org.br ${ }^{2}$ Programa de Pós-Graduação em Política Social, Universidade de Brasília.

${ }^{3}$ Departamento de Genética, Universidade Federal do Rio Grande do Sul.

\title{
A tese da judicialização da saúde pelas elites: os medicamentos para mucopolissacaridose
}

\author{
The thesis of judicialization of health care by the elites:
} medication for mucopolysaccharidosis thedication tor micopolysaccharidosis

\begin{abstract}
This paper evaluates the hypothesis that the judicialization of medicine for mucopolysaccharidosis in Brazil is an action promoted by economic elites. Previous studies upholding the thesis of judicialization by elites in the case of other types of medication that are more costly for the Unified Health Service are discussed. An analysis of all 196 processes containing information about judicial processes brought to court between February 2006 and December 2010 that ended by determining that the State should provide such medication free of charge to patients was conducted. There is evidence that attorneys' fees were covered by entities interested in the results of judicialization, such as the distributors or pharmaceutical industries. Patients may also be migrating for diagnosis and treatment to university centers that are a benchmark for medical innovation in the country, as the option for public health services is related to their higher technical and scientific capacity. Therefore, the resort to private lawyers, indicators of social exclusion based on the address of patients and the use of public health services, are not adequate class information to corroborate or refute the thesis of judicialization by the elites.
\end{abstract}

Key words Judicialization, Health policy, Mucopolysaccharidosis, Rare genetic diseases
Resumo O artigo avalia a hipótese de se a judicialização de medicamentos para o tratamento das mucopolissacaridoses no Brasil seria uma ação das elites econômicas. Debatem-se estudos prévios que defendem a tese da judicialização pelas elites em outros medicamentos. Discute-se, a metodologia desses estudos e as inferências dela derivadas e o respaldo empírico dessa tese no caso de um dos medicamentos judicializados de mais alto custo para o SUS. Foram analisados os 196 processos julgados entre fevereiro de 2006 e dezembro de 2010 que determinam a provisão gratuita dos medicamentos para mucopolissacaridoses pelo $\mathrm{Mi}$ nistério da Saúde. Há evidências de que os custos advocatícios sejam financiados por entidades interessadas nos resultados da judicialização, como as empresas distribuidoras ou indústrias farmacêuticas, de que pode haver migração dos pacientes para diagnóstico e tratamentos em centros universitários de referência para a inovação médica no país, e de que a opção por serviços públicos se dá por sua capacidade técnica e científica superior à de outras instituições. Logo, a advocacia privada, indicadores de exclusão social do local de residência dos pacientes e uso de serviços públicos não são informações de classe que corroborem ou refutem a tese da judicialização pelas elites.

Palavras-chave Judicialização, Política de saúde, Mucopolissacaridose, Doenças genéticas raras 


\section{Introdução}

Os estudos empíricos sobre a judicialização da saúde no Brasil indicam que medicamentos são o principal objeto de litígio nas cortes ${ }^{1,2}$. A política de assistência farmacêutica para a provisão gratuita de medicamentos no sistema de saúde brasileiro é guiada por princípios universalistas, e nem a legislação que regulamenta o direito à saúde, nem as políticas do Sistema Único de Saúde (SUS) focalizam o atendimento em função da classe social dos usuários ou estabelecem limites financeiros aos gastos com medicamentos em tratamentos. Há certos medicamentos, porém, que o SUS não oferece regularmente em seu dispensário. Em resposta a isso, alguns usuários acionam judicialmente o poder público a fim de obrigá-lo a prover esses medicamentos. Esse fato é particularmente importante no caso de medicamentos de alto custo, para os quais as despesas com o tratamento de um único indivíduo podem ser extremamente elevadas e nos quais a União é, geralmente, acionada.

Há várias razões para um medicamento não constar do dispensário regular do SUS, mas três delas merecem destaque. A primeira refere-se à administração de estoques: certos medicamentos requerem estocagem especial, outros permitem estocagem por períodos curtos apenas e alguns são consumidos apenas eventualmente e não justificam formação de reservas amplas. Há, além disso, erros no planejamento de estocagem e antecipação de demandas. As dificuldades em administrar esses estoques aliadas a demoras regulares na tramitação de processos de compra e redistribuição fazem com que a disponibilidade do medicamento seja comprometida. A segunda razão é a não inclusão do medicamento nas listas de dispensa, seja porque não se reconhece cientificamente a eficácia terapêutica do medicamento ou porque, apesar do reconhecimento científico, o trâmite de autorização pelo sistema de vigilância sanitária não foi concluído. A terceira razão é a recusa da distribuição em função da existência de potenciais substitutos com melhor relação custo-benefício (eficiência terapêutica), conhecida como a tese da racionalidade em saúde.

Os medicamentos para o tratamento das mucopolissacaridoses (MPS) são citados como um caso paradigmático para o estudo da judicialização da saúde ${ }^{3}$. Eles estão entre os mais caros no cenário da judicialização nacional e têm, cada um, sua produção monopolizada por uma única empresa farmacêutica, além de não terem sido incluídos na política de assistência farmacêutica do SUS até dezembro de 2011.
As MPS são doenças genéticas degenerativas raras causadas pela atividade deficiente de uma das enzimas envolvidas no catabolismo dos glicosaminoglicanos (antigamente denominados mucopolissacarídeos). São doenças crônicas, manifestam-se clinicamente na infância e reduzem significativamente a expectativa de vida. A maioria dos pacientes são crianças ou adolescentes. A população vivendo com MPS no Brasil é pequena. Há onze tipos de MPS, mas medicamentos para apenas três delas, a MPS I (laronidase), a MPS II (idursulfase) e a MPS VI (galsulfase). Os três medicamentos possuem registro na Agência Nacional de Vigilância Sanitária (Anvisa). Ensaios clínicos duplo-cegos randomizados contra placebo evidenciam benefícios e segurança com o tratamento com esses medicamentos, inclusive reversão parcial da sintomatologia preexistente, mas evidências consistentes sobre prevenção ou estabilização da doença são escassas. A magnitude dos benefícios, em termos de significância clínica, parece pequena ${ }^{4-6}$.

O objetivo deste estudo é verificar se a judicialização de medicamentos para o tratamento das MPS é um fenômeno de classe - mais especificamente, testar a hipótese se a judicialização seria promovida por elites econômicas. Ao fazer isso, o artigo debate diretamente estudos prévios que argumentam que a judicialização é um fenômeno de classes superiores. Discute-se, de um lado, a metodologia desses estudos e as inferências dela derivadas e, de outro, o respaldo empírico a essa tese no caso de um dos medicamentos judicializados de mais alto custo para o SUS.

O SUS é regido por princípios universalistas e não discrimina o atendimento segundo a classe econômica dos usuários. Com ou sem judicialização, pessoas com capacidade de pagamento podem obter tratamento gratuito sem que isso viole as regras do sistema. Este estudo não tem como meta discutir se a judicialização agride princípios filosóficos de justiça como equidade, mas sim se há evidências razoáveis de que a judicialização de medicamentos para MPS estaria concentrada em estratos sociais elevados.

A maior parte dos estudos brasileiros sobre judicialização de medicamentos enfoca os aspectos negativos dessa prática para a regulação da política pública, para a justiça distributiva e para o funcionamento da democracia ${ }^{1}$. Diversos estudos defendem a tese de que a judicialização de medicamentos rompe princípios de equidade em saúde, pois ocorre predominantemente entre as classes da população que seriam capazes de adquirir os medicamentos solicitados ${ }^{7-10}$. Para fins 
de simplificação, denominamos esse argumento de tese da judicialização pelas elites, pois cálculos a partir dos dados de Vieira e Zucchi ${ }^{9}$ indicam que o valor médio dos gastos públicos com medicamentos ofertados em decorrência de processos judiciais era de R $\$ 5.152$ em 2005, montante que corresponde a cerca de quatro vezes o rendimento médio do quinto mais rico da distribuição de rendimento mensal per capita brasileira naquele ano.

${\text { Chieffi e } \text { Barata }^{10} \text {, Vieira e Zucchi }}^{9}$, por exemplo, defendem essa tese analisando resultados empíricos com base em dois indicadores de classe social: a contratação de advogados privados e a baixa vulnerabilidade ou exclusão social - vulnerabilidade medida pelo Índice Paulista de Vulnerabilidade Social (IPVS) e exclusão pelo Índice de Exclusão Social do Município de São Paulo (Iex) da área geográfica onde vivem os demandantes. A capacidade de aquisição privada dos medicamentos seguramente seria mais bem medida por indicadores diretos de renda, mas os dois estudos precisariam lançar mão dessa estratégia, pois nos dados de que dispunham não havia registro de rendimentos familiares dos demandantes.

As conclusões de Chieffi e Barata ${ }^{10}$ retiradas da análise desses indicadores são particularmente fortes. Sobre a natureza profissional dos advogados, afirmam: o fato de essas ações geralmente serem ajuizadas por advogados particulares mostra que os pacientes arcaram com os custos dessa representação e em princípio poderiam adquirir os medicamentos solicitados. Sobre o local de residência, concluem: [...] a classificação de residência dos pacientes pelo IPVS reforça a hipótese de que a interferência do Poder Judiciário na política de saúde rompe o princípio da equidade ao favorecer as demandas dos que menos necessitam, em detrimento daqueles que só podem contar com o sistema público de saúde, ampliando a inequidade já existente.

A tese da judicialização das elites também é defendida por Borges e Ugá ${ }^{8}$ em função do tipo de serviço de saúde utilizado para as prescrições: ressalte-se que existem evidências de que o gasto público decorrente das demandas judiciais por medicamentos é altamente regressivo, uma vez que favorece os segmentos de mais alta renda da população, pois grande parte das prescrições nos processos judiciais é proveniente dos serviços privados de saúde. Essa é uma interpretação de resultados de terceiros que, no estudo original de Pereira et al. ${ }^{7}$, recebe um tratamento diferente e muito menos polarizado: Considerando que foi observado um predominio de prescrições originárias no serviço privado, pode-se afirmar que há um público 'misto', capaz de custear consultas e eventuais exames, diretamente ou por meio da medicina suplementar, mas que recorre ao Sistema Único de Saúde para a obtenção dos medicamentos.

Os estudos mencionados analisam a judicialização de uma ampla gama de medicamentos e agem com prudência no que diz respeito à generalização de seus resultados, sendo explícitos ao tratar suas conclusões como restritas a áreas geográficas bem definidas. Neste artigo, temos a situação inversa: cobertura nacional, mas restrita a um grupo menor de medicamentos e de requerentes. Todavia, esses estudos têm em comum o uso de evidências empíricas para fundamentar uma discussão normativa que se aplicaria a todo o SUS, especificamente sobre os efeitos da litigância sobre a justiça distributiva do sistema de saúde. É por esse motivo que atenção especial deve ser dada à metodologia utilizada nos estudos, não só no que diz respeito à possibilidade de generalização de resultados de pesquisa para vários medicamentos em todo o país, mas também no que se refere à validade dessas conclusões para as áreas geográficas analisadas e sua extrapolação para o restante do Brasil.

\section{Metodologia}

A hipótese de que a judicialização da demanda por medicamentos para MPS é restrita às elites econômicas foi testada a partir de uma análise de processos judiciais determinando a provisão gratuita dos medicamentos pelo Ministério da Saúde (MS). Nos arquivos do MS foram recuperados 196 processos conduzidos entre fevereiro de 2006 e dezembro de 2010, correspondendo à demanda de 195 indivíduos (não há ações coletivas, a maioria dos processos é individual, mas há cinco processos com dois autores e um indivíduo com dois processos). Não houve exclusão de processos da análise. A Consultoria Jurídica do MS considera o total de 196 casos o universo da judicialização dos três medicamentos para MPS (laronidase, indursulfase e galsulfase) que alcançou o ministério até dezembro de 2010 e, por isso, os dados são tratados como tendo natureza censitária. Não foram consultados dados sobre ações judiciais interpostas contra outros entes da Federação em que a União não constasse como corré.

O MS possui sistema próprio de registro e sistematização dos dados de processos judiciais 
em que participa como réu, mas esse registro não é feito para fins de pesquisa. Por esse motivo, optou-se por não utilizar os registros administrativos e realizar a coleta diretamente nos documentos. Para assegurar confidencialidade, os processos foram lidos na íntegra em sala reservada no MS e os dados foram registrados em formulário eletrônico com validação em três rodadas de préteste, cada uma testando 40 (20\% do total) processos, totalizando 120 testes $(61 \%)$. Ao final do levantamento, para controlar a qualidade da coleta, a equipe contrastou os dados coletados nos processos com os registrados pelo MS. Em caso de discordância entre os dois registros, a verificação recorria aos processos originais.

O teste de uma hipótese de judicialização pelas elites requer uma definição de elite econômica. A tendência geral dos estudos de estratificação é utilizar algum tipo de linha de riqueza construída a partir de dados de rendimentos familiares ${ }^{11}$. Os processos judiciais, no entanto, não registram informação sobre renda dos litigantes, o que impede o uso desse tipo de linha e indica que o dado não foi considerado na formação do convencimento do juiz. Problema similar foi enfrentado por dois outros estudos brasileiros sobre judicialização da saúde ${ }^{9,10}$. Na impossibilidade de obter informações sobre renda, optou-se pelo uso de indicadores indiretos de status socioeconômico.

Indicadores indiretos não seriam recomendáveis para o propósito de defender a tese da judicialização pelas elites, mas prestam-se mais adequadamente para questionar essa tese. Os estudos anteriores identificam a classe social dos usuários do SUS utilizando: i) a natureza profissional dos advogados; ii) indicadores de vulnerabilidade (IPVS) ou exclusão social (Iex); e iii) o tipo de serviço de saúde. O uso de indicadores indiretos merece uma discussão mais pormenorizada.

No que se refere à natureza profissional dos advogados, não é simples assumir que a contratação de um advogado privado caracterizaria a posição de elite do requerente. O pressuposto que justificaria a validade desse tipo de indicador seria o de que a barreira de entrada para a advocacia privada é alta, isto é, que os custos de acesso a um advogado privado seriam proibitivos para grande parte da população. Este não parece ser um pressuposto razoável. Primeiro, porque, embora seja correto assumir que os honorários de advogados de elite possam ser altos, assim como o acesso à medicina privada, a advocacia privada também pode ter custos absorvíveis por muitas pessoas fora das elites. Segundo, porque esses custos nem sempre são cobertos pelos de- mandantes. Há casos em que governos estaduais e municipais firmam convênios com advogados privados, subsidiando os custos de serviços de assistência judiciária. A Procuradoria-Geral do Estado de São Paulo, por exemplo, firmou convênios desse tipo com a seção local da Ordem dos Advogados do Brasil a partir de 1997 (a um custo médio por ação de R\$238). Terceiro, porque não há informações nos processos sobre quem arca com os honorários advocatícios. A judicialização é um palco com vários atores interessados, entre eles a indústria farmacêutica, que têm motivos econômicos para subsidiar a advocacia privada ${ }^{2,7,9,12,13}$. Isso pode indicar interesse de elites (indústria farmacêutica) na judicialização, mas de modo algum permite inferir que os demandantes dos medicamentos no SUS fazem parte dessas elites.

Por outro lado, se a natureza profissional dos advogados não é um indicador seguro da condição de elite, ela pode ser um indicador de posicionamento no outro extremo da escala social: dada a insuficiência de defensores públicos no Brasil, seria pouco plausível supor que as elites recorrem a eles para defender seus interesses, até porque para recorrer à defensoria pública o indivíduo atesta em documento de fé pública sua hipossuficiência de renda. Assim, quem recorre à defensoria pública muito provavelmente não pertenceria à elite, mas quem recorre à advocacia privada pode ou não pertencer à elite. Em consonância com esse raciocínio, neste estudo optamos por tratar o uso de advogados públicos (categoria que inclui defensores e procuradores) como indicador de que os demandantes não pertencem à elite e o recurso a advogados privados como indicador de pertencimento a qualquer classe social.

Resultados obtidos a partir de indicadores sintéticos de vulnerabilidade e exclusão montados por agregação espacial também podem ser questionados. Essas medidas se alinham mais a indicadores de pobreza do que de riqueza: seus construtos revelam hipossuficiência de recursos, não afluência. Tome-se, por exemplo, o IPVS, um índice sintético construído por análise fatorial, que atribui grande carga à porcentagem no setor censitário de responsáveis por domicílios com rendimentos de até três salários mínimos. Os valores do IPVS permitem classificar os indivíduos como vulneráveis ou não, mas não permitem inferir que indivíduos não vulneráveis podem dispensar a assistência do SUS, quanto menos concluir com segurança que o atendimento de não vulneráveis ameaça a equidade de um sistema de saúde. 
Além disso, o IPVS e o Iex são indicadores sintéticos obtidos pela combinação de variáveis que dificilmente poderiam ser justificadas em uma classificação mais rigorosa de indivíduos em estratos sociais elevados, tais como idade e níveis de alfabetização. Finalmente, o uso de um agregado espacial de domicílios - e não de dados individualizados - é justificável, dada a indisponibilidade de melhores informações, mas sua validade seria afetada no caso de migração para tratamento. Neste artigo, não são utilizados indicadores de renda dos indivíduos demandantes, mas atenção é dada à migração para tratamento por meio da relação entre a distribuição espacial das instituições de saúde e o local de origem de seus pacientes.

Tratar prescrições realizadas em instituições de saúde privadas como indicador de renda alta pressupõe que os custos de acesso à medicina privada são muito elevados. Tal como no caso da advocacia privada, essa suposição não é razoável. Antes de tudo é preciso diferenciar os custos de um diagnóstico eventual e do tratamento regular. $\mathrm{O}$ uso regular de tratamento na medicina privada pode ser inviável para muitas pessoas, mas é plausível aceitar que nas circunstâncias particulares envolvidas na judicialização uma fração grande da população seria capaz de pagar para obter prescrições. Se, por um lado, é pouco provável que a população pobre tenha condições de pagar por diagnósticos privados, por outro, o fato de uma pessoa poder arcar com os custos de uma consulta médica não permite caracterizá-la como "segmento de mais alta renda da população" ou da elite. Tampouco seria possível tratar como não sendo da elite alguém que recorre à medicina pública. Isso porque parte da medicina de alta qualidade que as elites demandam é também encontrada em centros de referência, como os hospitais universitários, onde grande parte da inovação tecnológica em saúde está concentrada.

No caso específico das MPS, o diagnóstico depende de exames que não são disponibilizados pelo SUS. A Rede Hospitalar Sarah realiza esses exames somente para os seus pacientes, apesar de pertencer ao SUS. Assim, os profissionais que atendem os demais pacientes no Brasil organizaram, na maioria das vezes com o apoio financeiro das indústrias farmacêuticas envolvidas na produção e comercialização da laronidase, idursulfase e galsulfase, e mesmo de agências públicas de fomento científico, um sistema informal que possibilita o diagnóstico, sem custos para o paciente e para o médico assistente. Nesse contexto, é possível esperar que mesmo a população de baixa renda utilize a medicina privada para obter prescrições para os medicamentos das MPS, assim como também é possível considerar que pacientes da elite utilizem os hospitais universitários para o diagnóstico de doenças raras.

Neste artigo, analisa-se a distribuição das instituições de saúde responsáveis pelo tratamento dos usuários do SUS por tipo de instituição (pública geral, pública universitária ou privada). A razão para isso não é associar o uso de serviços públicos à posição de classe das pessoas, mas avaliar se a dispersão do tratamento das MPS está relacionada à pesquisa médica. Como ocorre comumente em levantamentos documentais judiciais, um problema enfrentado foi o de ausência de uniformidade nos termos usados para registro de informação nos processos - por exemplo, nomes completos ou abreviados de instituições de saúde. Para lidar com isso, a equipe de levantamento coletou os dados tais como registrados e uma segunda equipe se encarregou de sua verificação junto às instituições de saúde e de sua posterior normalização.

\section{Resultados}

No Ministério da Saúde, a judicialização de laronidase, idursulfase e galsulfase, medicamentos para as doenças do grupo das MPS, foi feita por 195 indivíduos beneficiados por 196 decisões judiciais entre 2006 e 2010, que juntos consumiram R\$ 219.664.476,05. A determinação judicial de aquisição de medicamentos para MPS implica, portanto, uma compra média por ação superior a 1,1 milhões de reais. O paciente típico (modal) das ações é uma criança com menos de 10 anos, que se mantém em tratamento entre um e três anos, encaminhada por um hospital público e defendida por um advogado privado. Um em cada vinte pacientes foi a óbito durante o tratamento. Uma única empresa distribuidora, a Uno Healthcare, responde por $97 \%$ dos valores transacionados. Esses processos foram distribuídos a 104 juízes, a maioria deles julgando apenas um processo.

Observou-se uma distribuição expressivamente concentrada de advogados. Um único advogado é responsável pelos processos de 70 (36\%) pessoas, as quais, em conjunto, correspondem a um gasto de mais de 77 milhões de reais. Constam 40 advogados nos processos, mas apenas três deles estão relacionados a mais da metade de todos os gastos do governo com a judicialização de medicamentos para MPS e qua- 
se $60 \%$ das doses adquiridas. Esses três são advogados privados.

No outro extremo da distribuição estão os 21 advogados responsáveis pelo processo de apenas um paciente. Eles correspondem a mais da metade da lista de advogados, porém estão relacionados a apenas $10 \%$ das quantidades adquiridas e menos de $15 \%$ de todo o gasto despendido. Nesse grupo, encontram-se, com alguma frequência, defensores e procuradores públicos, embora sejam ainda minoria. Do total de 195 indivíduos, 155 foram representados por advogados privados, 20 por defensores e procuradores públicos, e, sobre 21 indivíduos, não há informações. Nenhum dos defensores e procuradores públicos defendeu mais de quatro indivíduos, mas entre os advogados privados há pelo menos seis que trabalharam para cinco ou mais pacientes.

Não há correlação forte entre a dispersão geográfica dos advogados e a incidência das MPS no país. Observou-se que, entre os defensores, procuradores públicos e advogados privados conduzindo processos de até quatro pessoas, a judicialização geralmente ocorre na mesma unidade da Federação onde o paciente recebe tratamento. Esse quadro, porém, é diametralmente oposto no caso dos advogados privados que concentram grandes volumes de processos. Nestes, os processos de pacientes residindo na unidade da Federação onde os advogados atuam são minoria.

Existem informações sobre os serviços de saúde de origem de 89\% (174) dos indivíduos. No total, 53 instituições são listadas. A maior parte dessas instituições trata de um ou dois indivíduos. No entanto, há seis instituições tratando de 10 ou mais indivíduos, o que corresponde a $41 \%$ dos pacientes com serviço de saúde conhecido. As instituições estão distribuídas em todo o Brasil, mas há concentração de indivíduos sendo tratados em serviços de saúde das metrópoles das regiões Sul e Sudeste, onde se localizam centros de referência universitários para a pesquisa sobre as MPS.

Para uma população em que predominam crianças e jovens, observa-se migração muito elevada, tanto em relação à cidade de origem no início do processo judicial e à cidade de tratamento quanto em relação à naturalidade do paciente e cidade de origem. Apenas 56 (32\%) dos 177 indivíduos cujo processo trazia informação completa viviam na mesma cidade antes do tratamento com os medicamentos (início do processo judicial) e no momento do acesso ao serviço de saúde. Essa migração, porém, já havia sido iniciada: 42 (44\%) dos 96 indivíduos com informação sobre natura- lidade já haviam migrado de sua cidade natal à época da abertura do processo.

Dos 178 pacientes cujos serviços de saúde foram completamente identificados, 144 (81\%) recorreram a hospitais públicos. Destes, 83 (58\%) foram a hospitais universitários, e a maioria dos hospitais públicos atendendo os demais pacientes estava de algum modo relacionada à pesquisa em doenças genéticas. Naqueles hospitais públicos atendendo 10 ou mais indivíduos predomina a situação em que um único médico responde pela indicação do medicamento para todos os pacientes e não raro um único advogado é responsável pela maioria dos processos.

\section{Discussão}

Os medicamentos para MPS têm alto custo e seus mercados de produção e distribuição são monopolizados por poucas empresas Os grandes volumes financeiros movimentados fazem com que os resultados da judicialização de medicamentos sejam objeto de interesse dessas empresas. Uma interpretação dos resultados obtidos nesta pesquisa não pode ignorar as potenciais consequências desses interesses.

Há uma altíssima concentração de casos em poucos advogados privados. Por outro lado, não há forte correlação entre o local de residência ou a origem dos pacientes e o local de atuação desses advogados concentradores. É pouco provável que a contratação atomizada de advogados privados por uma população geograficamente dispersa gerasse um padrão distributivo desse tipo, o que sugere a existência de algum tipo de rede que coloca em contato pacientes e advogados. Não há evidência empírica suficiente para fazer qualquer afirmação segura sobre a organização dessa rede, mas considerando que o volume médio de uma ação ultrapassa o custo normal de um advogado privado na ordem de milhares de vezes, uma hipótese que merece ser cogitada é a de que essa rede seria financiada pela indústria farmacêutica ou pelos distribuidores dos medicamentos.

Os indícios da existência de uma rede potencialmente financiada por empresas interessadas nos resultados da judicialização são o primeiro elemento que desafia a noção de que apenas as elites recorreriam a advogados privados na judicialização. O segundo elemento é o custo relativamente baixo da contratação privada de alguns advogados. Os estudos que utilizaram o recurso à advocacia privada como indicador de classe $e^{9,10}$ presumiram que esse custo é excessivo para as 
pessoas comuns, mas o exemplo dos convênios realizados pelo Governo do Estado de São Paulo desde 1997 mostra que isso pode não ser sempre verdade. Diante desses dois elementos, o mais razoável seria reconhecer que o recurso à advocacia privada não é um indicador adequado de classe, ou seja, não permite corroborar ou refutar a tese da judicialização pelas elites.

Observam-se níveis elevados de migração entre os pacientes, que, na sua maioria, são crianças e adolescentes. Há uma concentração de pacientes em poucos serviços de saúde que tratam das MPS e estes estão, majoritariamente, localizados em metrópoles. É possível que essa migração ocorra em função de busca por diagnósticos e tratamento. Esse não é um tipo convencional de migração e, portanto, não se deve esperar que a distribuição espacial dos migrantes nas cidades de destino siga os padrões de outros tipos de migração. Primeiro, porque é razoável aceitar que se essa migração não é entendida como temporária, ao menos deve ser tratada como excepcional. Fatores como a proximidade das instituições de saúde ou a conveniência da coabitação com outras famílias podem ser determinantes da localização espacial desses migrantes.

Não é provável que os padrões típicos de segregação espacial por classe nas metrópoles brasileiras sejam integralmente observados nesse tipo de migração. Logo, é questionável o procedimento metodológico de identificação da posição de classe desses indivíduos a partir de seu local de residência, como fizeram outros estudos ${ }^{9,10}$. Ainda que o problema de usar um índice sintético agregado de pobreza, vulnerabilidade ou exclusão para identificar posições mais altas na estratificação social fosse resolvido, restariam dúvidas sobre a validade da presunção de que existiria uma correlação estreita entre local de residência e classe social em uma população com um padrão migratório muito peculiar. Neste caso tão específico parece mais prudente assumir que o local de residência não é um bom indicador de nível de renda das pessoas e, portanto, não permite fazer afirmações seguras sobre o padrão distributivo da judicialização de medicamentos, em particular, e da saúde, em geral.

A predominância de pacientes recorrendo a hospitais públicos não pode ser tomada como um indicador de que se trata de pacientes de baixa renda. A forte concentração de pacientes em alguns hospitais e o fato de esses hospitais concentradores serem também instituições de pesquisa em genética clínica sugerem que as razões para recorrer aos serviços gratuitos não são es- tritamente econômicas. Há três hipóteses para essa concentração da prescrição médica em centros públicos de pesquisa. A primeira é que essas instituições são a porta de entrada para a pesquisa sobre inovações tecnológicas no país, em particular para os medicamentos. Em consequência, são espaços privilegiados para a prescrição de novas tecnologias em saúde. A segunda hipótese é que a genética clínica para doenças genéticas raras ainda não foi regulamentada como uma subespecialidade médica no SUS, portanto, é nos hospitais universitários que se concentra a assistência a doenças genéticas, como as MPS. Por fim, a terceira hipótese é que a indústria farmacêutica é uma das principais financiadoras da pesquisa em genética clínica no país, e os centros listados são, na sua maioria, financiados por uma ou mais empresas que produzem ou distribuem os medicamentos judicializados.

Sejam quais forem os motivos, nos processos de pacientes dos hospitais mais frequentes predominam poucos médicos e poucos advogados, o que evidencia a formação de redes para a judicialização dos medicamentos para as MPS, tal como observaram outros estudos sobre judicialização de outros medicamentos ${ }^{2,7,9,12,13}$. É possível, portanto, que a direção da causalidade seja do hospital para a judicialização, ou seja, é justamente porque alcança o hospital público de pesquisa que o paciente passa a integrar a rede mais ampla de interesses dentro da qual a judicialização ocorre. A complexidade de motivos determinando o uso de hospitais públicos desafia a possibilidade de usar o pagamento pelos serviços de saúde como medida de posição social de classe do indivíduo.

\section{Conclusões}

A base argumentativa que motiva as críticas à judicialização é a de que ela é conduzida por elites e isso ameaçaria o princípio da equidade no SUS. Esse argumento ignora o problema lógico de que as políticas universais não discriminam usuários com base em características individuais: pobres e ricos são igualmente protegidos pelo SUS. Se este fosse um sistema focalizado seria adequado entender que, pelo princípio da diferença rawlsiano, o atendimento de qualquer indivíduo que não aquele em piores condições feriria a equidade. Porém, para um sistema de saúde guiado por princípios universalistas, esse argumento é falacioso: para um julgamento da justiça de uma demanda em saúde não importa a origem de clas- 
se do indivíduo, mas sim a avaliação de quais demandas protegem necessidades de saúde não satisfeitas.

O objetivo deste artigo não foi discutir se a judicialização fere princípios filosóficos de justiça como equidade em uma política universalista de saúde, até mesmo porque a judicialização pode ser um instrumento legítimo para a promoção da igualdade, mas avaliar se há evidências razoáveis de que seriam as elites que judicializam medicamentos para as MPS no Brasil. Uma análise da metodologia utilizada por estudos que defendem a tese da judicialização pelas elites e as evidências empíricas obtidas nesta pesquisa sobre os medicamentos para as MPS indicam que os fundamentos empíricos sobre os quais essa tese poderia se fundamentar não são seguros:

i. A advocacia privada não é bom indicador de posição de classe. Primeiro, porque os custos advocatícios podem estar sendo financiados por entidades interessadas nos resultados da judicialização - empresas distribuidoras, por exemplo. Segundo, porque mesmo que o financiamento fosse feito pelos próprios usuários, sob as circunstâncias particularíssimas da busca por tratamento em saúde, os custos de advocacia privada podem ser uma barreira para pessoas muito pobres, mas não necessariamente para uma grande massa da população.

ii. O local de residência dos pacientes não é um bom indicador de classe. A migração em busca de tratamento médico é um tipo muito singular de migração, por isso não se pode presumir que os padrões médios de segregação espacial por classe típicos de metrópoles brasileiras se repliquem entre esses migrantes. O posicionamento desses indivíduos nas cidades para garantir tratamento pode ter relação com fatores não estritamente econômicos.

iii. O uso de serviços públicos ou privados de saúde não é um bom indicador de classe. Os tratamentos de alto custo judicializados muitas vezes estão relacionados à medicina altamente especializada; esta, por sua vez, é encontrada em serviços públicos de saúde dedicados à pesquisa científica e à inovação tecnológica, como os hospitais universitários. Neste caso, mesmo os indi- víduos mais ricos teriam razões para preferir serviços públicos.

A conclusão geral a que se pode chegar é a de que, usando as metodologias de outros estudos e os dados até o momento disponíveis sobre a judicialização de medicamentos para a MPS, não é possível refutar nem corroborar a tese da judicialização pelas elites. Não há como dizer que a judicialização é uma questão de classe. O que se coloca é se essa conclusão pode ser extrapolada para a judicialização de outros medicamentos ou mesmo outros tipos de judicialização da saúde.

Uma extrapolação desse tipo só será segura com o acúmulo de evidências em outros estudos e, por isso, qualquer generalização feita neste momento deve ser tomada com cautela. No entanto, como é razoável aceitar que os mecanismos que motivam e possibilitam a judicialização dos medicamentos para as MPS sejam compartilhados por outros tipos de judicialização, alguma extrapolação pode ser feita: indicadores de natureza profissional dos advogados, local de residência e uso de serviços médicos públicos ou privados não são indicadores sólidos para sustentar teses definitivas sobre os padrões distributivos de renda do público que judicializa medicamentos no Brasil.

Mas como o que motiva a avaliação da tese da judicialização pelas elites são preocupações de natureza moral quanto à ameaça da judicialização ao igualitarismo em saúde, as conclusões deste debate devem ir além da avaliação dessa tese. Se há um problema distributivo relevante no debate sobre judicialização, ele não parece estar na origem de classe dos usuários que recorrem ao Judiciário, mas i) na composição de interesses de laboratórios e empresas distribuidoras de medicamentos de alto custo cuja eficácia clínica ainda está sob avaliação; ii) nas dificuldades do Estado em administrar a política de assistência farmacêutica quanto aos critérios de incorporação tecnológica, transparência e publicidade dos atos; e iii) nas dificuldades do Estado em administrar uma política farmacêutica quando instrumentos típicos de administração - planejamento, compras em escala, controle de estoques, chamadas de preços - não podem ser usados em decorrência de determinações judiciais. 


\section{Colaboradores}

M Medeiros, D Diniz e IVD Schwartz participaram igualmente de todas as etapas de elaboração do artigo.

\section{Agradecimentos}

O projeto foi financiado pela Organização PanAmericana da Saúde, Agradecemos a colaboração de Vanessa Carrião, Renata Brito, Natália Peres e João Neves com a coleta e o processamento dos dados. Gisélia Ferreira, Valéria F. Paiva, Suelen G. Anjos, Eduardo B. Felizola e Wilza C. Fávila foram interlocutoras na etapa de pesquisa de campo, a quem agradecemos as autorizações para a pesquisa empírica.

\section{Referências}

1. Ventura M, Simas L, Pepe VLE, Schramm FR. Judicialização da saúde, acesso à justiça e a efetividade do direito à saúde. Physis 2010; 20(1):77-100.

2. Machado MA de Á, Acurcio F de A, Brandão CMR, Faleiros DR, Guerra Júnior AA, Cherchiglia ML, Andrade EIG. Judicialização do acesso a medicamentos no Estado de Minas Gerais, Brasil. Rev Saude Publica 2011; 45(3):590-598.

3. Boy R, Schwartz IVD, Krug BC, Santana-da-Silva LC, Steiner CE, Acosta AX, Ribeiro EM, Galera MF, Leivas PGC, Braz M. Ethical issues related to the access to orphan drugs in Brazil: the case of mucopolysaccharidosis type I. J Med Ethics [periódico na internet]. 2011 Jan 25 [acessado 2011 set 1]; 37(4):233-239. Disponível em: http://jme.bmj.com/ content/early/2011/01/24/jme.2010.037150.abstract.

4. Muenzer J, Wraith JE, Beck M, Giugliani R, Harmatz P, Eng CM, Vellodi A, Martin R, Ramaswami U, Gucsavas-Calikoglu M, Vijayaraghavan S, Wendt S, Puga AC, Ulbrich B, Shinawi M, Cleary M, Piper D, Conway AM, Kimura A. A phase II/III clinical study of enzyme replacement therapy with idursulfase in mucopolysaccharidosis II (Hunter syndrome). Genet Med 2006; 8(8):465-473.

5. Wraith JE, Clarke LA, Beck M, Kolodny EH, Pastores GM, Muenzer J, Rapoport DM, Berger KI, Swiedler SJ, Kakkis ED, Braakman T, Chadbourne E, WaltonBowen K, Cox GF. Enzyme replacement therapy for mucopolysaccharidosis I: a randomized, doubleblinded, placebo-controlled, multinational study of recombinant human alpha-L-iduronidase (laronidase). J Pediatr 2004; 144(5):581-588.

6. Harmatz P, Giugliani R, Schwartz I, Guffon N, Teles EL, Miranda MCS, Wraith JE, Beck M, Arash L, Scarpa M, Yu ZF, Wittes J, Berger KI, Newman MS, Lowe AM, Kakkis E, Swiedler SJ; MPS VI Phase 3 Study Group. Enzyme replacement therapy for mucopolysaccharidosis VI: a phase 3, randomized, double-blind, placebo-controlled, multinational study of recombinant human $\mathrm{N}$-acetylgalactosamine 4-sulfatase (recombinant human arylsulfatase B or rhASB) and follow-on, open-label extension study. I Pediatr 2006; 148(4):533-539.

7. Pereira JR, Santos RI dos, Nascimento Junior JM do, Schenkel EP. Análise das demandas judiciais para o fornecimento de medicamentos pela Secretaria de Estado da Saúde de Santa Catarina nos anos de 2003 e 2004. Ciênc Saude Colet. 2010; 15(Supl. 3):3551-3560.

8. Borges D da CL, Ugá MAD. Conflitos e impasses da judicialização na obtenção de medicamentos: as decisões de 1a instância nas ações individuais contra o Estado do Rio de Janeiro, Brasil, em 2005. Cad Saude Publica 2010; 26(1):59-69.

9. Vieira FS, Zucchi P. Demandas judiciais e assistência terapêutica no Sistema Único de Saúde. Rev Assoc Med Bras 2009; 55(6):672-683.

10. Chieffi AL, Barata RB. Judicialização da política pública de assistência farmacêutica e eqüidade. Cad Saude Publica 2009; 25(8):1839-1849. 
11. Medeiros M. The rich and the poor: The construction of an affluence line from the poverty line. So-

12. cial Indic Res 2006; 78(1):1-18.

Chieffi AL, Barata R de CB. Ações judiciais: estratégia da indústria farmacêutica para introdução de novos medicamentos. Rev Saude Publica 2010;

13. 44(3):421-429.

Messeder AM, Osorio-de-Castro CGS, Luiza VL. Mandados judiciais como ferramenta para garantia do acesso a medicamentos no setor público: a experiência do Estado do Rio de Janeiro. Cad Saude Publica 2005; 21(2):525-534.

Artigo apresentado em 09/10/2011

Aprovado em $25 / 11 / 2011$

Versão final apresentada em 13/12/2011 